\title{
New modeling approach of laser communication in constellation and through atmospheric disturbances
}

\author{
Hachemi Chenina', Djamel Benatia², M'Hamed Boulakroune ${ }^{3}$ \\ ${ }^{1}$ Electronic Department, Faculty of Technology, University of Batna 2, Algeria \\ ${ }^{2}$ Laboratoire d'Électronique Avancée (LEA), Electronic Department, Faculty of Technology, University Batna 2, Algeria \\ ${ }^{3}$ Laboratory of Electrical Engineering Polytechnic Constantine, Electrical and Automatic Department, National \\ Polytechnic School of Constantine, Algeria
}

\begin{tabular}{l} 
Article Info \\
\hline Article history: \\
Received Jan 10, 2021 \\
Revised Apr 29, 2021 \\
Accepted Jun 24, 2021 \\
\hline
\end{tabular}

Keywords:

Atmospheric turbulence Inter-satellite lasercommunication Optical communication Optical satellites constellation Pointing systemes

\begin{abstract}
Laser communication between satellites in the constellation and from the satellites to ground stations offers a gigantic data rate for the users. This principal advantage drives telecom companies to develop this technology to use it like a carrier signal, the most disadvantage of this technology is the need to very complicated pointing systems between the transmitter and the receiver due to a very small beam divergence, continually moving of satellites in orbits and the distance between the satellites (tens of thousands of kilometers). The laser beam suffers continuously from several factors like atmospheric turbulences, internal and external vibrations. All these factors lead to an increase in the bit errors rate and cause degradation in the communication quality. This paper deals with a new method of modelisation of external effects in transmission of signal light from a ground station to the satellite through atmospheric disturbances. Indeed, an in-depth investigation, of the influences of satellite vibrationsinlaser signal transmission between satellites constellation, has been conducted by studying the effect of the intensity of vibrations on the optical signal amplitude. Some solutions are proposed to improve the efficiency of optical satellites communications.
\end{abstract}

This is an open access article under the $\underline{C C B Y-S A}$ license.

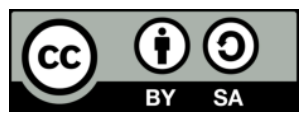

\section{Corresponding Author:}

Hachemi Chenina

Electronic Department, Faculty of Technology

University of Batna 2

05000 Batna, Algeria

Email: chenina31@yahoo.fr

\section{INTRODUCTION}

Using laser beam in optical satellites communication is thought to be the most important technology because of its advantages vs the radio frequency links, such as license-free frequency band, rapid and simple implementation, adaptable transmitter and receiver design, and extremely reliable sensitive data transfer [1], Installation costs are modest, energy requirements are modest, the beam size is narrow, and the bandwidth is wide. This approach allows people to communicate without interfering from electromagnetic fields [2], [3]. Given all these advantages, we use laser beams in satellite communications systems (up-link, down-link, constellation of satellites and inter-satellite link). Data is sent from one spacecraft to another orbiting in the same or separate orbits using free space/vacuum as the propagation medium and optically modulated carrier signals in inter-satellite optical wireless communication (IsOWC) connections. M. Singh, and J. Malhotra [4] inter-satellite connections (ISLs) are critical for maintaining a modest number of ground stations. Without the use of ground stations, traffic can be diverted from spacecraft to another one using ISLs. The iridium system is an operating LEO satellite networks of RF-ISLs [5]. The laser beam on its way from the transmitter to the 
receiver suffers from many effects that increase the BER and thus decrease the efficiency of the communication system. These effects include atmospheric disturbances in the atmosphere that affect the ascending signal to the satellite, when the signal reaches the satellite and moves between the constellations of satellites, it is exposed to many factors that affect the quality of communication, this factors include internal and external vibrations.

The air turbulence due to changes occurred in temperature and atmospheric pressure. According to the theory of Kolmogorov successive energy, it produces cells in the formation of a turbulent, called "vortices", which have different sizes and refractive indices [6]. S. Liu, j. Yang, X. Guo, et al. [7] reports an algorithm of arranging ISLs in the laser/radio hybrid network. A review on different difficulties looked by free space optical (FSO) communications system for inter-satellite links has been deliberated in [8]. C. Wang et al. [9], the author present detailed analysis of ISL observations. In particular, he implement observation model refinements and highlight the contribution of ISL measurements. M. Toyoshima [10] introduces the trends of space laser communications for constellations. G. Curzi et al. [11] the author aim at the perspective of matching technical needs and technology availability for large constellations, while providing at the same time a detailed survey of the up coming satellite constellations, By introducing a representative list of RSSC missions, I. Sanad et al. [12] provides an overview of what is currently understood about satellites networks configurations and orbit types used for satellite remote sensing constellation (RSSC) missions. The work in [13] establish a model for a trusted device quantum key distribution (QKD) spacecraft in low earth orbit (LEO) and test its efficacy in various network configurations examine the importance of inter-satellite QKD connections in addition. T. Savitri et al. [14] describe a method to address a complex coverage area by using sparse satellite constellation design with a limited coverage capability. The results of [15] show how the onaxis scintillation index of annular Gaussian beams and BER change in vertical directions for laser satellite communications networks. The performance of optical wireless system that employs M-ary PPM of a Gaussian beam, in terms of BER when the optical wireless system is operated in an anisotropic nonKolmogorov turbulent atmosphere are specified in Y. Ata et al [16]. For GEO and MEO laser satellite connections, a modern and theoretical time series synthesizer of the log-amplitude of the laser signal due to scintillation is proposed in [17]. H. Singh et al. [18] presents turbulent atmospheric channels with zero and non zero boresight pointing errors and elucidates the effect of phase noise on performance of FSO communication. M. Yasser et al. [19] presents the FSO link system and channel models. Mathematical analysis and close-form expressions for SER and outage probability are proposed.

In this work, for the first time, we report new modeling approach of laser communication in constellation and through atmospheric disturbances in the communication bitween ground station and satellite through Atmospheric week and strong turbulances and bitween the satellites in constellations including a modelation of internal vibrations of the satellite sub-systems in a constellations and studing the effects of this vibrations on the quality of the optical signal (SNR, BER). With the suggestion of some possible solutions to reduce the negative effects of these vibrations.

The paper is organized as follows, various atmospheric effects like, beam broadening, turbidity, astronomical refraction and scintillation are discussed in section 2, atmospheric turbulence model are presented in section 3, laser communications in constellations of satellies under the effects of the internal satellites vibrations are discussed in section 4, simulation results are discussed in section 5, a proposed solutions to mitigate the undesirable effects of vibrations are presented in section 6 , finally, the conclusion is presented in section 7.

\section{LASER COMMUNICATION THROUGH ATMOSPHERIC CHANNEL}

\subsection{Description of the atmospheric turbulence}

There are many previous studies that dealt in depth with the chemical components of the atmosphere and the effects of wind and sea-level rise on the spread of electromagnetic waves and laser beams [2], [20]. The atmosphere is a complicated structure with a high level of sophistication. It is clear that the whole range of processes that constitute the atmosphere and its relationship with laser radiation must be known before useful optical devices for activity in the atmosphere can be planned. The atmosphere chemical composition has been well explained in the reference [20], [21]. The atmospheric turbidity reported in [22]. References [23]-[26] explain the astronomical refraction and air turbulence.

\subsection{The effect of turbulence on the optical beam}

Most research employed the "frozen" turbulence hypothesis to simulate laser radiation in the atmosphere [23]. The approach includes the assumption that the time change at any place is produced by the uniform beam movement of the entire atmosphere due to prevailing wind [23], [27], [28]. The evolution of turbulence in the atmosphere causes changes in the internal structure of the atmosphere. The evolution of turbulence through time causes changes in the internal structure of the atmosphere, which are ignored. 


\subsubsection{Beam broadening}

Let us consider the beam size in free space as $W$ and the intensity at the distance $L$ is given by the formula: (see Figure 1)

$$
I(x, \rho)=I_{0}\left(\frac{W_{0}}{W}\right)^{2} \exp \left[-\frac{2 \rho^{2}}{W^{2}}\right]
$$

where $W$ is the beam width in free space and $W_{\mathrm{b}}$ is the beam width in turbulence [23], [29], $I_{o}$ is the intensity of the incident beam on the beam axis, $W_{\mathrm{o}}$ is the beam size at the transmitter, $\rho$ is the transverse distance from the beam axis

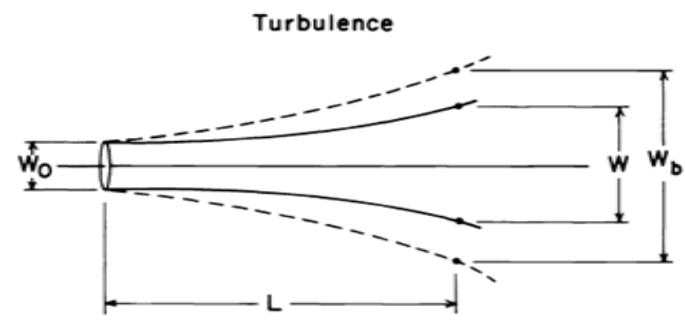

Figure 1. Beam spread

The beam size $W$ for a collimated beam in free space is given by:

$$
W^{2}=W_{0}^{2}+\left(\frac{2 L}{k W_{0}}\right)^{2}
$$

The beam size $W_{\mathrm{b}}$ in the turbulence is given by:

$$
W_{b}^{2}=\frac{W^{2}}{2 \int_{0}^{\infty} \exp \left[-t^{2}-(1 / 2) D_{s} t^{5 / 3}\right] t d t}
$$

\subsubsection{Spatial coherence}

The loss of spatial coherence on the laser beam is another significant consequence of disturbance in the open skies. Nonlinear phase variations can result from uneven refractive indices at wide scales. Thereby reducing the coherence of the wave front radiation. The structure function of the phase fluctuation $D_{s}\left(\rho_{1}, \rho_{2}\right)$ is defined as [23], [30]:

$$
D_{s}\left(\rho_{1}, \rho_{2}\right)=E\left[\left|s\left(\rho_{1}\right)-s\left(\rho_{2}\right)\right|^{2}\right]
$$

where $\rho_{1}$ and $\rho_{2}$ are position vectors in the plane of observation across the beam and $s($.$) is the phase at that$ point. For weak turbulence, [31] gives a simple result for the phase structure function

$$
D_{s}(0, \rho)=2.91 b_{1} C_{n}^{2} z \rho^{5 / 3}
$$

where the value of $b_{1}$ ranges from 1.0 for a plane wave to 0.375 for a spherical wave.

The phase correlation between the two points $\rho_{1}$ and $\rho_{2}$ of the wave-front decreases with the distance $\rho=\left|\rho_{1}-\rho_{2}\right|$. For plane waves or spherical waves, the coherence can be expressed as:

$$
\gamma(z, \rho)=\exp \left[-\left(\frac{\rho}{\rho_{0}}\right)^{5 / 3}\right]
$$

where $\rho_{0}$ is the radius of phase coherence which is presented as:

$$
\rho_{0}=\left(b_{2} C_{n}^{2} k^{2} z^{2}\right)^{-3 / 5}
$$


where $b_{2}=1.45$ for a plane wave and 0.55 for a spherical wave. When $\rho \geq \rho_{0}$, the random phase angle variation is greater than $\pi$, It is thought that the wave-front has lost its spatial coherence [23], [30].

\subsection{Effect of turbidity on optical radiation}

Light is absorbed and scattered by atmospheric elements such as gases and dust. As a result, the turbidity of the atmosphere must be considered when designing an effective laser device, and must consider the reduction of the direct beam energy when the light propagates in the atmosphere.

\subsubsection{Absorption and scattering loss}

Water, carbon dioxide, and ozone molecules are the primary atmospheric absorbers [32]. The absorption of light by the atmosphere is a wavelength-dependent phenomenon. In databases like MORTRAN, the wavelength dependence of attenuation under various weather situations is usually provided [33].

\section{ATMOSPHERIC TURBULENCE MODEL}

Turbulence is caused by random fluctuations in the atmosphere's refractive indices. The temperature difference between the atmosphere, the ground, and the ocean produces the change in refractive index, which causes airflow and wind to enter the top layer of the atmosphere. Temperature, pressure, length of wave, and humidity all affect the fixed refractive index $n_{0}[34]$.

$$
n_{0} \approx 1+\frac{77 \mathrm{p}}{T}\left[1+\frac{7.53 \cdot 10^{-3}}{\lambda^{2}}-7733 \frac{\mathrm{q}}{T}\right] 10^{-6}
$$

where $q$ is specific humidity $\left(\mathrm{gm}^{3}\right), \lambda$ is wavelength, $T$ is temperature $(\mathrm{K}), p$ is air pressure (millibars).

When dealing with random motion of the refractive indices, Kolmogorov's model is commonly applied. The refractive indices, according to this idea, is the sum of fixed and variable components [35]. $n_{T}(r)=n_{0}+n(r)$; Where $n_{0}$ is the median indice of refraction, $r$ is the position in space, and $n(r)$ is the random component produced by the spatial variation of pressure, temperature and humidity. The basic statistical parameter is the spatial cross-correlation of the refractive index, which is defined as [36]:

$$
\Gamma_{n}\left(r_{1}, r_{2}\right)=\mathrm{E}\left[n\left(r_{1}\right), r(2)\right]
$$

where E[ ] Signifies the expected value.

The power spectral density is the three-dimensional Fourier transform of the spatial correlation of the refractive index, given by the references [37]-[39] as:

$$
\Phi(K)=0.033 C_{n}^{2} K^{-11 / 3}
$$

where $C_{n}^{2}$ is the indice of refraction [17], and $K$ satisfies the inequality $2 \pi / L_{0}<K<2 \pi / l_{0} ; l_{0}$ is the internal limit and $L_{0}$ is the external limits.

The inertial range of the Kolmogorov spectrum defined by (11) is limited by internal and external constraints. $C_{n}^{2}$ Differs between $10^{-13} \mathrm{~m}^{-2 / 3}$ for severe disturbances to $10^{-17} \mathrm{~m}^{-2 / 3}$ of weak disturbances. Empirical data forms the basis of a model for determining $C_{n}^{2}$ values, such as the sum of highly correlated indices used in reference [36].

$$
C_{n}^{2}(h)=A \exp \left(-\frac{h}{H_{A}}\right)+B \exp \left(-\frac{h}{H_{B}}\right)+C h^{10} \exp \left(-\frac{h}{H_{C}}\right)+D \exp \left(-\frac{\left(h-H_{D}\right)^{2}}{2 d_{C}^{2}}\right)
$$

where $A$ is the coefficient of turbulence intensity on the surface (boundary layer), $H_{A}$ is the altitude of its $1 / \mathrm{e}$ attenuation, $\mathrm{B}$ and $\mathrm{HB}$ denote the troposphere disturbance, $\mathrm{C}$ and $\mathrm{HC}$ denote the highest point of the troposphere disturbance level, D and HD define an isolated turbulent layer, and dc is the thickness of the layer.

A random Maxwell wave equation is used to compare the index of refraction function with the electromagnetic model. Rytov's approach achieves the simplification of Maxwell's equations [40], [41]. The field is supposed to be a combination of the free space field and the random complex amplitude transmittance representing the field disturbance.

$l_{0}<\sqrt{Z \lambda}<L_{0}$ where $\lambda$ is the wavelength and the turbulent coherence diameter $d_{0}$ can be approximated by reference [40], [41]. $d_{0}<\sqrt{Z \lambda}$. We think of the downlink as a plane wave because most of 
the path of the laser beam travels through space and is only affected by turbulence when it approaches earth. References [31], [40], [41] give the covariance of plane waves as:

$$
\sigma_{X}^{2}(Z)=0.56\left(\frac{2 \pi}{\lambda}\right)^{7 / 6} \int_{0}^{Z} C_{n}^{2}(x)(Z-x)^{5 / 6} d x
$$

The density distribution function of $X$ is normal, as shown [40]:

$$
f_{X}(X)=\frac{1}{\sqrt{2 \pi \sigma_{x}}} \exp \left(-\frac{X-E[X]^{2}}{2 \sigma_{X}^{2}}\right)
$$

Normalized received power is related to logarithmic amplitude $X$ :

$$
I=\exp (2 X-2 E[X])
$$

After studying the spread of the laser beam in the atmosphere and analyzing the effects of atmosphere components on the light signal, the signal now reaches the satellite and travels through a constellation of satellites to reach the satellite located above the receiver. In this part, we will analyze the transmission of this signal between the satellites, and the various effects of the light beam.

\section{NETWORK COMMUNICATION MODEL}

A satellite constellation is a set of related satellites that operate together to form a network. The LEO constellation of satellites is configured to act as a network to provide more coverage [42], [43]. The considered optical inter-satellite network model includes transmitter satellite, repeater satellites, and receiver satellite all networked together [44].

\subsection{Signal model}

We arrive at a model in this subsection that connects the laser signal transmitted by the first spacecraft in the constellation to the signal received by the last spacecraft. The optical signal obtained by the constellation's second spacecraft is:

$$
\mathrm{PR}=\mathrm{K} \cdot \mathrm{L}
$$

where

$$
K=\eta_{T} \cdot \eta_{R}\left(\frac{\lambda}{4 \cdot \pi \cdot Z}\right)^{2} \cdot G_{R} \cdot G_{T} \cdot P_{T}
$$

The optical signals received by satellite $(n+1)$ in the network is [45]

$$
P_{R}(n+1)=\prod_{i=1}^{n} K_{i} L_{i}
$$

with:

$$
K_{i}=G_{i} \eta_{T i} \eta_{R i+1}\left(\frac{\lambda}{4 \pi Z_{i}}\right)^{2} G_{T i} G_{R i+1} i=2,3, \ldots, n,
$$

Where $G_{i}$ is the gain of the network's spacecraft relays $i . P_{T}$ is the optical strength of the emitter, $Z i$ is the spacing among spacecraft $i$ and $i+1 . n$ represent the number of spacecraft in the constellation, $\eta_{T i}$ is the photonics efficiency of the emitter spacecraft $i$ in the constellation and $\eta_{R i}$ is the photonics efficiency of the reception spacecraft $i$ in the constellation.

The targeting loss factor is determined as [46]:

$$
L_{i}=\exp \left(-G_{T_{i}} \theta_{i}^{2}\right)
$$

where $\theta_{i}$ is the angle of radial pointing inaccuracy. The transmitter gain of satellite $i$ in the network is: 


$$
G_{T i}=\left(\frac{\pi D_{T i}}{\lambda}\right)^{2}
$$

where $D_{T i}$ is the size of the emitter aperture in the $i$ satellite in the constellations.

The receiver gain of the $i$ satellite in the satellites constellation is [45]:

$$
G_{R i}=\left(\frac{\pi D_{R i}}{\lambda}\right)^{2}
$$

where $D_{R i}$ is the size of the receiver aperture in the $i$ satellite in the constellations. if all satellites are the same

$$
G_{T i}=G_{T j}=G_{T i} \forall i, j
$$

The optical signals received by satellite $(n+1)$ in the network is

$$
P_{R}(n+1)=\exp \left(-G_{T} \sum_{i=1}^{n} G_{T i} \theta_{i}^{2}\right) \prod_{i=1}^{n} K_{i}
$$

From (24)

$$
P_{R}(n+1)=\exp \left(-G_{T} X\right) \prod_{i=1}^{n} K_{i}
$$

where

$$
X=\sum_{i=1}^{n} \theta_{i}^{2}
$$

Each $\theta_{i}$ contains two normal processes (azimuth and elevation), so $X$ includes $2 n$ normal processes. The process distribution composed of the sum of squares of normal processes with equal variances is chisquare. If all satellites are the same, the standard deviation of the radial pointing error angle of all satellites is equal to [47]:

$$
\sigma_{i}=\sigma_{j}=\sigma_{1}=\sigma_{X} \forall i, j
$$

Therefore $X$ is a chi-square distribution with probability density:

$$
f(X)=a X^{-1+n} \exp \left(-\frac{X}{2 \sigma_{1}^{2}}\right) U(X)
$$

where

$$
a=\frac{1}{\left(\sigma_{1} \sqrt{2}\right) m^{2 n} \Gamma(\mathrm{n})}
$$

The gamma function is:

$$
\Gamma(\mathrm{x})=\int_{0}^{\infty} \mathrm{t}^{\mathrm{x}-1} \exp (-\mathrm{t}) \mathrm{dt} 1 \leq \mathrm{x} \leq 2
$$

The average optical power received in the $n$ satellite is

$$
S=a \prod_{i=1}^{n} K_{i} \int_{0}^{\infty} x^{-1+n} \exp \left(-G_{T} x \frac{x}{2 \sigma_{1}^{2}}\right) d x
$$

After solving (30):

$$
S=\frac{\prod_{i=1}^{n} K_{i}}{\left(G_{T} 2 \sigma_{1}^{2}+1\right)^{n}}
$$

\subsection{Performance models}

The SNR model is [47]: 


$$
S N R=\int_{0}^{\infty} \ldots \int_{0}^{\infty} \frac{\left[R P_{R}\left(\theta_{1} \ldots \theta_{n}\right)\right]^{2}}{\sigma_{N T}^{2}\left(\theta_{1} \ldots \theta_{n}\right)} f\left(\theta_{1}\right) \ldots f\left(\theta_{n}\right) d \theta_{1} \ldots d \theta_{n}
$$

where $R$ is the response of the detector at the receiver satellite.

To simplify the (32), We suppose that the primary source of noise is in the constellation's final satellite. Then (32) becomes:

$$
S N R \approx \frac{R^{2} P_{T}^{2}}{\sigma_{N 2}^{2} G_{2}^{2}} \int_{0}^{\infty} \ldots \int_{0}^{\infty}\left[K_{2}^{2 n} \exp \left(-2 G_{T} \sum_{i=1}^{n} \theta_{i}^{2}\right)\right] f\left(\theta_{1}\right) \ldots f\left(\theta_{n}\right) d \theta_{1} \ldots d \theta_{n}
$$

Repeating the same procedure in (12) to (18) for (31)

$$
S N R \approx \frac{R^{2} P_{T}^{2}}{\sigma_{N 2}^{2} G_{2}^{2}} \frac{K_{2}^{2 N}}{\left(1+4 G_{T} \sigma_{1}^{2}\right)^{n}}
$$

The noise-to-signal ratio NSR to obtain a closed form [47]:

$$
N S R=\frac{\sigma_{N 2}^{2} G_{2}^{2}}{R^{2} P_{T}^{2}} \int_{0}^{\infty} \ldots \int_{0}^{\infty}\left[\frac{1+K_{2}^{2} \exp \left(-2 G_{T} \theta_{n}^{2}\right) \prod_{i=1}^{n-1} K_{i+1}^{2} \exp \left(-2 G_{T} \sum_{i=1}^{n-1} \theta_{i+1}^{2}\right)}{K_{2}^{2 n} \exp \left(-2 G_{T} \sum_{i=1}^{n} \theta_{i}^{2}\right)}\right] f\left(\theta_{1}\right) \ldots f\left(\theta_{n}\right) d \theta_{1} \ldots d \theta_{n}(35)
$$

Repeating the procedure in (35):

$$
N S R=\frac{\sigma_{N 2}^{2} G_{2}^{2}}{R^{2} P_{T}^{2}}\left[\frac{1}{K_{2}^{2 n}\left(1-4 G_{T} \sigma_{1}^{2}\right)^{n}}+\cdots+\frac{1}{K_{2}^{2}\left(1-4 G_{T} \sigma_{1}^{2}\right)}\right]
$$

In (35) provides us another factor for analyzing constellation efficiency.

\section{RESULTS AND DESCUSSIONS}

In this section, we give some results based in a parameters showen in Table 1. From the Figure 2, we can assume that the spatial coherence decreases when the altitude of the transmitter increases and decreases also with the strongest of the turbulence, for a $C_{n}^{2}=10^{-9}$ for weak turbulence the spatial coherence is greater than the strong turbulence with $C_{n}^{2}=10^{-7}$; so the altitude from the sea level and the strongest of the turbulence degrade the coherence of the propagating wave front. This leads to deterioration in the receiver, the beam pattern begins to decompose into different regions of high and low intensity. Absence of beam efficiency at the receptor can result in areas of spontaneous variability with low signal strength for sufficiently long path lengths and high optical turbulence amplitude, resulting in severe signal attenuation.

Table 1. Parameters used in this analyse

\begin{tabular}{lcc}
\hline \multicolumn{1}{c}{ Parameter Name } & Parameter Symbol & Parameter Value \\
\hline Link range & $L$ & $\approx 8 \times 10^{2} \mathrm{Km}$ \\
Optical received signal & $P_{\mathrm{R}}$ & - \\
Distance between the satellites & $Z$ & $40,000 \mathrm{Km}$ \\
Electronicbandwidth & $B$ & $2 \mathrm{GHz}$ \\
Transmitter/receiveroptics efficiency & $\eta_{T}, \eta_{R}$ & $80 \%$ \\
Wavelength & $\Lambda$ & $0.635 \mu \mathrm{m}$ \\
Turbulence force & $C_{n}^{2}$ & $10^{-7}$ \\
Altitude & $A$ & - \\
Received light intensity probability & $P_{w}(I)$ & - \\
Number of satellites in the constellation & $N$ & 20 \\
The radial pointing error angle's & $\sigma_{i}$ & - \\
The size of the receiver aperture in the $i$ satellite in the constellations & $D_{R i}$ & $0.2 \mathrm{~m}$ \\
\hline
\end{tabular}

Figure 3 illustrates how the magnitude of disturbance $C_{n}^{2}$ varies depending on the channel's different parameters, such as wind velocity, height, and environmental conditions. The $C_{n}^{2}$ was estimated between 0 and $20 \mathrm{~km}$ in this study and not greater since the value of $C_{n}^{2}$ is minimal at high latitudes. The intensity force is calculated using various altitudes and wind speeds. Wind speeds shear at the ground, affecting the atmosphere's refractive index and resulting in substantial disturbance output. Low turbulence is defined as a wind speed of $9 \mathrm{~m} / \mathrm{s}$, whereas moderate turbulence is defined as a wind speed of $18 \mathrm{~m} / \mathrm{s}$. This graph clearly 
shows that the highest value of $C_{n}^{2}$ is located near to the surface, and that as altitude grows, its value decreases, and this negatively affects the quality of the spread of the light signal.

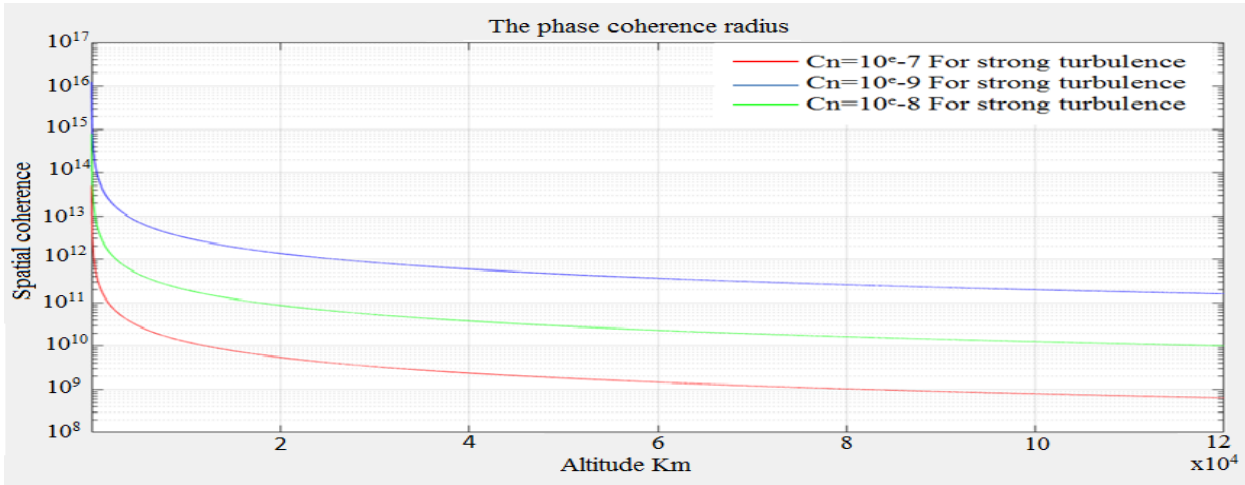

Figure 2. Spatial coherence vs. altitude (Km) and turbulence

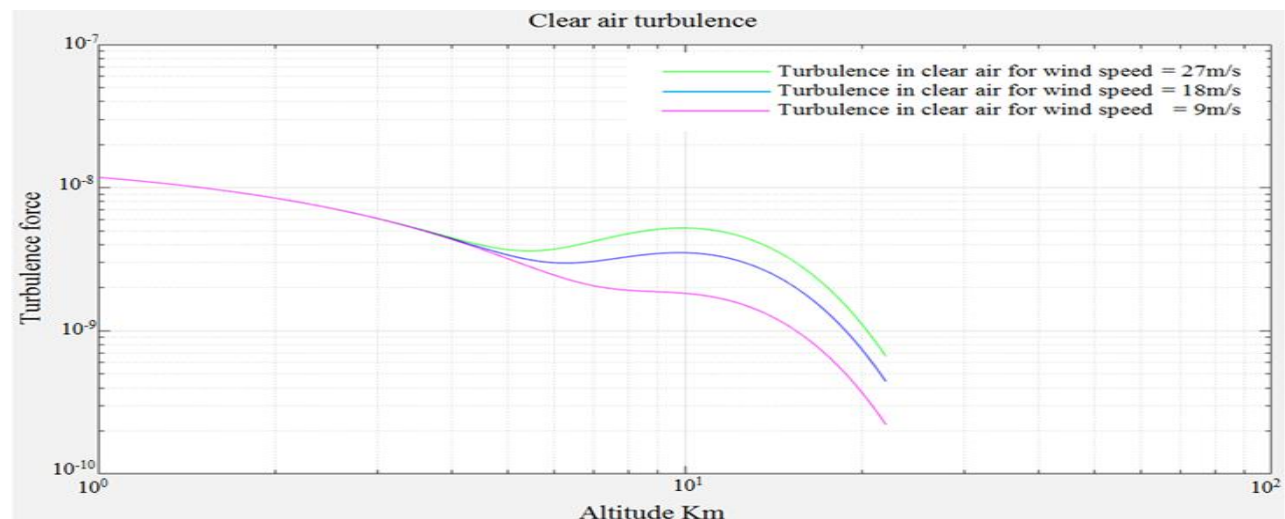

Figure 3. The force of turbulence vs. the altitude and the wind speed

From the Figure 4, we notice that scintillation (log-irradiation) is completely related to the angle of Zenith as shown in Figure 4 so any change, even very small, causes a complete loss of optical radiation and thus a explains the basic advantages of the use of lasers in communication. This is the great resistance against interference, which gives great safety to transmit the signal from the transmitter to the receiver. This result also explains the need for a very accurate tracking system that ensures connectivity in the event of movement and continuous vibration of the satellites.

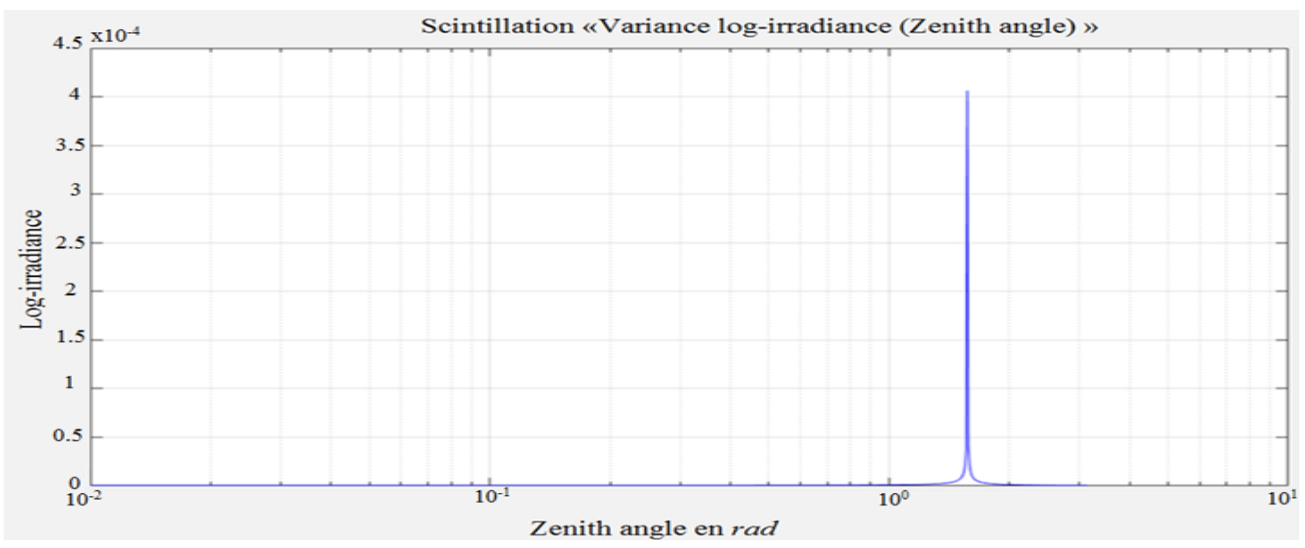

Figure 4. Log-irradiance as a function of zenith angle 
From Figure 5, we notice that the wavelength considerably affects the scintillation the increase in the wavelength causes a decrease in the scintillation and the decreasing in the wavelength causes an increasing in the scintillation. We also note that the wind speed affects scintillation, if the wind speed increases, the scintillation decreases and if the wind speed decreases, the value of the scintillation increases. This is due to the effect of wind speed on the strength of the air turbulence. Log-irradiance has a good value at a wavelength of less than $100 \mu \mathrm{m}$, and after the wavelength $1 \mathrm{~mm}$ it becomes negligible and close to zero.

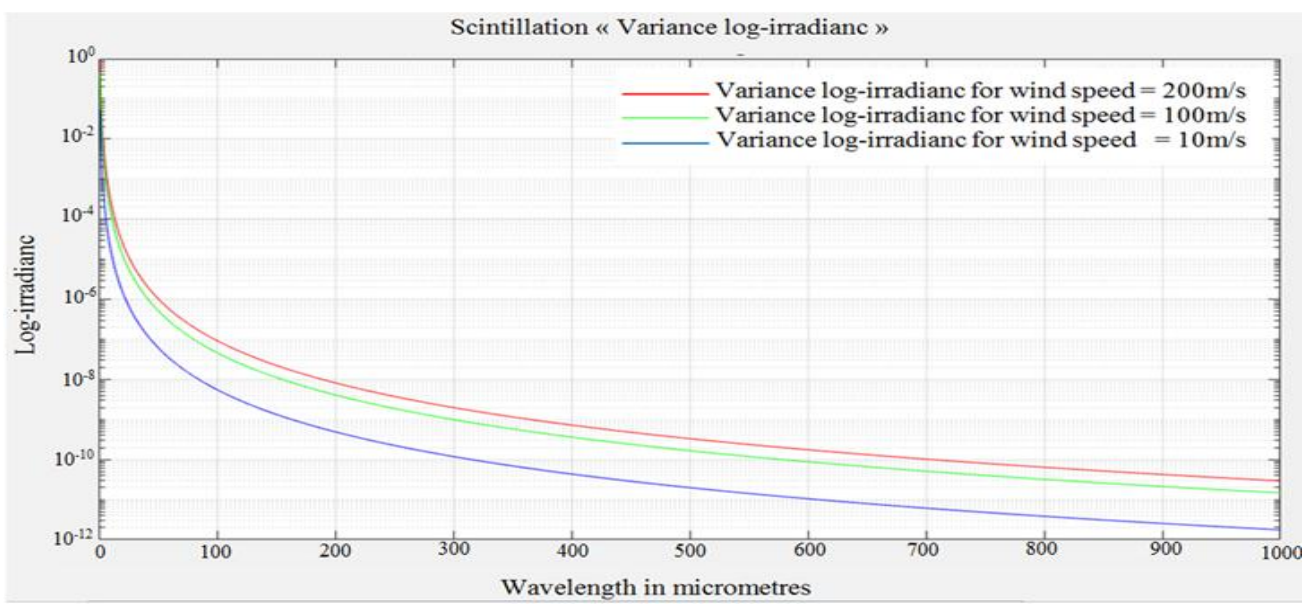

Figure 5. Log-irradiance vs. wavelength variance "Yura and McKinley model"

The normalized NSR is seen in Figure 6 as a result of the vibration amplitude and the number of satellites in the constellation; in this case, the spacecrafts is 20. It is significant to mention that even in the absence of disturbances; NSR reduces as the constellation expands due to the aggregation of noise in the passes among spacecraft. The obtained laser light as a function of the vibration amplitude normalized to the square root of the transmitter gain and the number of spacecraft in the constellation can be seen in Figure 7 . Indeed, as the constellation grows, an increase in vibration intensity greatly reduces the obtained signal, which is due to the accumulation of vibrations from the first satellite to the last one.

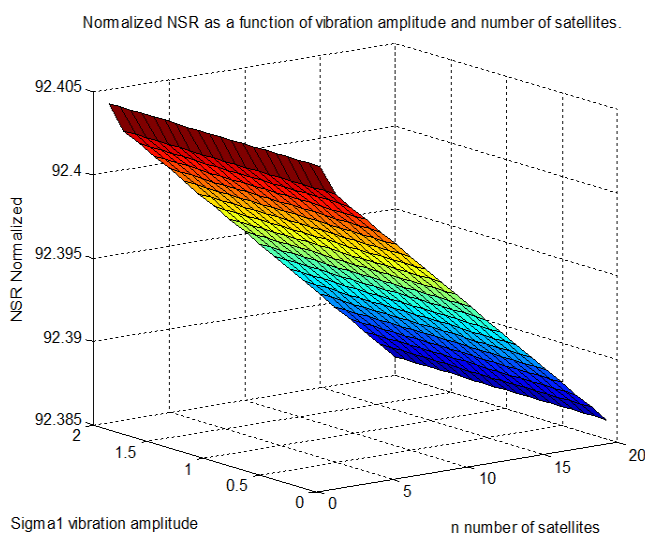

Figure 6. Normalized NSR as a function of vibration amplitude and number of satellites

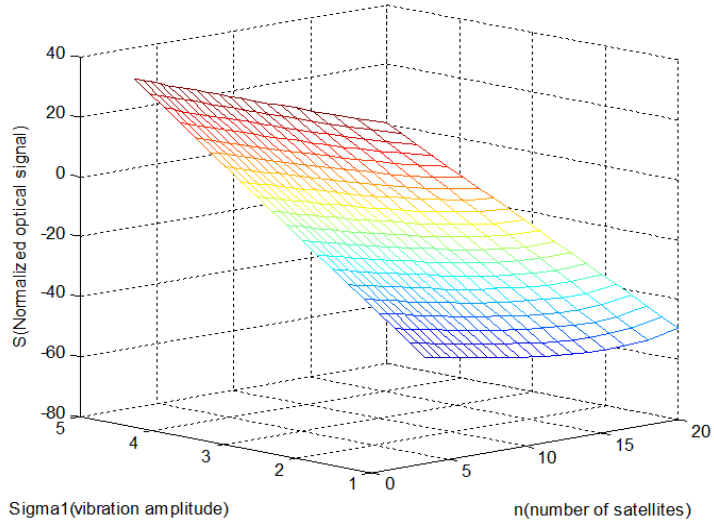

Figure 7. The received optical signal as a function of the vibration amplitude

\section{PROPOSED SOLUTIONS TO REDUCE THE EFFECTS OF ATMOSPHERIC TURBULENCE AND VIBRATIONS ON THE PERFORMANCE OF OPTICAL COMMUNICATION}

In this section we propose some solutions briefly to reduce the negative effects of vibrations and atmospheric turbulence, the proposed solutions to reduce the effects of satellite vibrations on the performance of optical communications are bandwidth adaptation, power control and channel diversity like what is mentioned in the references [44], Wavelength diversity and Temporal diversity are discussed in references 
[3], [6], [48], [49], Multiple emitters is mentioned in the reference [50]. In the following we will explain the most important solution which is: Vibration Isolator.

\subsection{Vibration isolator}

The techniques of micro-vibration isolation system can be classified into three main categories, passive, active, semi-active isolation [51]. A mechanical low-pass filter with a classic control mechanism is used in passive isolation system. Vibration control devices, force actuators, and displacement sensors are examples of active isolators. Passive isolators are used to minimize vibration distortion in high-frequency areas where the tuning mechanism's interference reduction capability is inadequate. Active isolators are used to dampen low frequency and high amplitude sounds that other devices are unable to block.

\section{DISCUSSION AND SUMMARY}

This article discusses the impact of pointing system vibration and atmospheric turbulence on the performance of optical communications in up-link and satellite networks. New modeling approach of laser communication in constellation and through atmospheric disturbances has been developed and compared with others proposed in the literature. We have demonstrated that The primary constellation performance parameter is the disturbance intensity, and the constellation size scale is a smaller one.

The results of our simulations show that scintillation (log-irradiation) is completely related to the angle of zenith, so any change, even very small, causes a complete loss of optical radiation, this result translates into one of the most important advantages of optical communication mentioned in previous studies [52], [53]. The wavelength and the wind speed considerably affect the scintillation in our modulation the increasing in the wavelength and the wind spead causes a decreasing in the scintillation and gradually approach a value of 0 , the reference [15] study also the affect of the propagation distance on the scintillation index. With an increasing propagation distance, the scintillation index is weakened by the loss of spatial coherence due to the focusing effect being.

The results of our simulations model show that the rise above the sea surface leads to a decrease in the strength of the air turbulence from $c_{n}^{2}=10^{-7.95}$ for the strong air turbulence to $c_{n}^{2}=10^{-8.7}$ for the medium air turbulence, regardless of the wind speed Until we reach a height of $400 \mathrm{~m}$, the force of the turbulence change as a function of wind speed also. Compared with result obtained by S. Arnon [35] the strength of turbulence decrease dramatically from $c_{n}^{2}=10^{-13}$ to $c_{n}^{2}=10^{-17}$ until the hight of $5000 \mathrm{Km}$ the wind speed affect also the strongest of turbulence. Finally, in light of these results, we suggested some solutions to reduce these effects of atmospheric turbulence and the internal satellite vibrations in constellation to increase the effectiveness of the laser communication system.

\section{CONCLUSION}

This paper has discussed the laser communication in the constellation of satellites and through atmospheric disturbance. A new modeling approach of laser communication was developed and simulated using Matlab software. The study carried out her can be the basis for future analyses of laser communication through the atmosphere and in the constellations of satellites. This analysis points out that the performance of the network is determined by the satellite that vibrates the most among the satellites in the network, the laser communication in space needs a very complicated pointing system.

\section{REFERENCES}

[1] S. Malik, P. K. Sahu, "Performance Analysis of Free Space Optical Communication System Using Different Modulation Schemes over Weak to Strong Atmospheric Turbulence Channels," Proceedings Optical and Wireless Technologies, Singapore, 2020, pp. 387-399, doi: 10.1007/978-981-13-6159-3_41.

[2] D. Dubey, Y. K Prajapati, R. Tripathi, "Error performance analysis of PPM-and FSK-based hybrid modulation scheme for FSO satellite downlink," Optical and Quantum Electronics, 2020, vol. 52, pp. 252-286, doi: 10.1007/s11082-020-02404-7.

[3] K. Sharma, S. K. Grewal, "Performance assessment of hybrid PPM-BPSK-SIM based FSO communication system using time and wavelength diversity under variant atmospheric turbulence," Optical and Quantum Electronics, vol. 52, pp. 1-25, 2020, doi: 10.1007/s11082-020-02547-7.

[4] M. Singh, J. Malhotra, "Modeling and Performance Analysis of 400 Gbps CO-OFDM Based Inter-satellite Optical Wireless Communication (IsOWC) System Incorporating Polarization Division Multiplexing with Enhanced Detection," Wireless Personal Communications, vol. 111, pp. 495-511, Mar 2020, doi: 10.1007/s11277-019-068705 . 
[5] M. Roth, H. Brandt, H. Bischl, "Implementation of a geographical routing scheme for low Earth orbiting satellite constellations using intersatellite links," International Journal of Satellite Communications and Networking, vol. 39, no. 1, pp. 92-107, 2021, doi: 10.1002/sat.1361.

[6] J. Y. Choi, W. H. Shin, S. K. Han "Turbulence induced fading mitigation in satellite optical communications using a single subcarrier time delay diversity," Optics Communications, vol. 477, no. 126360, pp. 1-6, 2020, doi: 10.1016/j.optcom.2020.126360.

[7] S. Liu, j. Yang, X. Guo, L. Sun, "Inter-satellite link assignment for the laser/radio hybrid network in navigation satellite systems," GPS Solutions, vol. 24, no. 49, pp. 1-14, 2020, doi: 10.1007/s10291-020-0961-9.

[8] G. Tiwari, R. C. S. Chauhan "A Review on Inter-Satellite Links Free Space Optical Communication," Indian Journal of Science and Technology, vol. 13, no. 6, pp. 712-724, 2020, doi: 10.17485/ijst/2020/v13i06/147998.

[9] C. Wang, Q. Zhao, J. Guo, J. Liu, G. Chen, "The contribution of intersatellite links to BDS-3 orbit determination: Model refinement and comparisons," Journal of the Institute of Navigation, vol. 66, no. 1, pp 71-82, 2019, doi: 10.1002/navi.295.

[10] M. Toyoshima, "Recent Trends in Space Laser Communications for Small Satellites and Constellations, " Journal of Lightwave Technology, vol. 39, no. 3, pp. 693-699, 2021, doi: JLT.2020.3009505.

[11] G. Curzi, D. Modenini, P. Tortora, "Large Constellations of Small Satellites: A Survey of Near Future Challenges and Missions," Aerospace, vol. 7, no. 9, pp. 1-18, 2020, doi: 10.3390/aerospace7090133.

[12] I. Sanad, Z. Vali, D. G. Michelson, "Statistical Classification of Remote Sensing Satellite Constellations," IEEE Aerospace Conference, pp. 1-15, doi: AERO47225.2020.9172299.

[13] T. Vergoossen, S. Loarte, R. Bedington, H. Kuiper, A. Ling, "Modelling of satellite constellations for trusted node QKD networks," Acta Astronautica, vol. 173, pp. 164-171, 2020, doi: 10.1016/j.actaastro.2020.02.010.

[14] T. Savitri, Y. Kim, S. Jo, H. Bang, "Satellite Constellation Orbit Design Optimization with Combined Genetic Algorithm and Semi analytical Approach," International Journal of Aerospace Engineering, vol. 2017, ID. 1235692, pp. 1-17, 2017.

[15] H. Gerçekcioğlu, "Performance of annular beams in weak atmospheric turbulence for satellite laser communications," Optics Communications, vol 439, pp. 233-238, 2019, doi: 10.1016/j.optcom.2019.01.030.

[16] Y. Ata, Y. Baykal, M. C. Gökçe, "BER performance of M-ary pulse position modulated communication systems in anisotropic non-Kolmogorov turbulent atmosphere," Waves in Random and Complex Media, pp. 1-12, 2020, doi: $10.1080 / 17455030.2020 .1807072$.

[17] N. K. Lyras, C. I. Kourogiorgas, A. D. Panagopoulos, "Scintillation due to atmospheric turbulence on optical GEO and MEO satellite links: Time series synthesizer for system level simulations," International Journal of Satellite Communications and Networking, pp. 1-12, 2020, doi: 10.1002/sat.1383.

[18] H. Singh, A. S. Sappal, "Moment-Based Approach for Statistical and Simulative Analysis of Turbulent Atmospheric Channels in FSO Communication," IEEE Access, vol. 7, pp. 11296-11317, 2019, doi: 10.1109/ACCESS.2019.2892320.

[19] M. Yasser, T. Ismail, A. Ghuniem, "FSO Communication with Nonzero Boresight and Jitter over Málaga Atmospheric Turbulence," 22nd International Conference on Transparent Optical Networks (ICTON), 2020, pp. 15, doi: 10.1109/ICTON51198.2020.9203348.

[20] I. K. Larin, "Chemical Composition of the Middle Atmosphere and Its Changes in the 21st Century," Russian Journal of Physical Chemistry B, vol. 12, no. 6, pp. 1094-1098, 2018, doi: 10.1134/S1990793118060064.

[21] A.K. Majumdar, J. Ricklin, "Effects of the atmospheric channel on free-space laser communications," Proceedings SPIE 5892, Free-Space Laser Communications V, pp. 1-16, 2005, doi: 10.1117/12.617922.

[22] G. López, F. J. Batlles, "Estimate of the atmospheric turbidity from three broad-band solar radiation algorithms. A comparative study," Annales Geophysicae, vol. 22, no. 8, pp. 2657-2668, 2004, doi: 10.5194/angeo-22-2657-2004.

[23] Shaik, K. S, "Atmospheric propagation effects relevant to optical communications," The Telecommunications and Data Acquisition Report, pp. 180-200, 1988.

[24] H. Kaushal, G. Kaddoum, "Optical Communication in Space: Challenges and Mitigation Techniques," IEEE Communications Surveys \& Tutorials, vol. 19, no. 1, pp. 57-96, Firstquarter 2017, doi: 10.1109/COMST.2016.2603518.

[25] J. C. Owens, "Optical Refractive Index of Air: Dependence on Pressure, Temperature and Composition," Applied Optics, vol. 6, no.1, pp 51-59, 1967, doi: 10.1364/AO.6.000051.

[26] M. L. Wesely, E. C. Alcaraz, "Diurnal cycles of the refractive index structure function coefficient," Journal of Geophysical Research, vol. 78, no. 27, pp. 6224-6232, 1973, doi:10.1029/JC078i027p06224.

[27] V. Lavrinov, L. Lavrinova, "Statistical Properties of Phase Fluctuations of Optical Radiation Propagating through Atmospheric Turbulence," Russian Open Conference on Radio Wave Propagation RWP, 2019, pp. 205-208, doi: 10.1109/RWP.2019.8810362.

[28] R. S. Lawrence, J. W. Strohbehn, "A survey of clear-air propagation effects relevant to optical communications," Proceedings of the IEEE, vol. 58, no. 10, pp. 1523-1545, 1970, doi: 10.1109/PROC.1970.7977.

[29] A. Abdulsalam, "Free Space Optical Communications-Theory and Practices," Contemporary Issues in Wireless Communications, 1th ed. croatia: intech, pp. 159-212, 2014.

[30] X. Zhu, J. Kahn. "Communication techniques and coding for atmospheric turbulence channels," Journal Optical and Fiber Communications Reports, vol. 4, no. 6, pp. 363-405, 2007, doi: 10.1007/s10297-006-0077-1.

[31] V. E. Zuev, "Laser Beams in the Atmosphere,” New York, Consultants’ Bureau, 1982. 
[32] D. Anandkumar, R. G. Sangeetha, "A survey on performance enhancement in free space optical communication system through channel models and modulation techniques," Optical and Quantum Electronics, vol. 53, no. 5, pp. 139, 2021, doi: 10.1007/s11082-020-02629-6.

[33] F. G. Smith, J. S. Accetta, D. L. Shumaker, "The infrared \& electro-optical systems handbook: Atmospheric propagation of radiation," SPIE press, vol. 2, 1993.

[34] V. Kulikov, M. Vorontsov, "Analysis of the joint impact of atmospheric turbulence and refractivity on laser beam propagation," OSA Publishing, vol. 25, no. 23, pp. 28524-28535, 2017, doi: 10.1364/OE.25.028524.

[35] S. Arnon, et al, "Performance limitation of laser satellite communication due to vibrations and atmospheric turbulence: down-link scenario," International Journal of Satellite Communications and Networking, vol 21, no 6, pp. 561-573, 2003, doi: 10.1002/sat.769.

[36] S. Li, S. Chen, C. Gao, A. E. Willner, J. Wang, "Atmospheric turbulence compensation in orbital angular momentum communications: Advances and perspectives," Optics Communications, vol. 408, pp. 68-81, 2018, doi: 10.1016/j.optcom.2017.09.034.

[37] H. Yan, et al, "Propagation of Optical Coherence Vortex Lattices in Turbulent Atmosphere," Applied Sciences, vol. 8, no. 12, pp. 1-10, 2018, doi: 10.3390/app8122476.

[38] M. Al-gobi, D. Benatia, M. Bali "A hybrid algorithm for wave-front corrections applied to satellite-to-ground laser communication," Telecommunication Computing Electronics and Control TELKOMNIKA, vol.18, no.3, pp. 12591267, 2020, doi: 10.12928/telkomnika.v18i3.12960.

[39] F. Ö. Sayan, H. Gerçekcioğlu, Y. Baykal, "Multimode laser beam scintillations in weak atmospheric turbulence for vertical link laser communications," Waves in Random and Complex Media, pp. 1-13, 2020, doi: 10.1080/17455030.2020.1841331.

[40] N. S. Kopeika, "Sources of Optical Radiation, A System Engineering Approach to Imaging," SPIE Press Monograph, vol. PM38, 1998.

[41] L. C. Andrews, R. L. Phillips, "Optical Turbulence in the Atmosphere," Laser Beam Propagation through Random Media.2nd, Washington USA: SPIE Press, 2005, doi: 10.1117/3.626196.

[42] H. Ronald "Overview: Satellite Constellations," Journal of Space Operations \& Communicator, vol. 17, no. 2, pp. $1-16,2020$

[43] M. O. Kolawole, "Satellite communication engineering," CRC Press 2nd edition, New York, Mar. Dek, ch 5, 2002.

[44] S. Arnon, N. S. Kopeika, "Laser satellite communication network-vibration effect and possible solutions," Proceedings of the IEEE, vol. 85, no. 10, pp. 1646-1661, Oct. 1997, doi: 10.1109/5.640772.

[45] S. Arnon, N. S. Kopeika, S. Rotman, "Performance limitations of free-spaceoptical communication satellite networks due to vibrations: Direct detection digital mode," Optical Engineering, vol. 36, no. 11, pp. 3148-3157, 1997, doi: 10.1117/1.601553.

[46] C.-Chen, C. S. Gardner, "Impact of random pointing and tracking errors on the design of coherent and incoherent optical intersatellite communication links," IEEE Transactions on Communications, vol. 37, no. 3, pp. 252-260, March 1989, doi: 10.1109/26.20099.

[47] S. Arnon, N. S. Kopeika, "The performance limitations of free space optical communication satellite networks due to vibrations-analog case," Proceedings of 19th Convention of Electrical and Electronics Engineers, 1996, pp. $287-$ 290, doi: 10.1109/EEIS.1996.566951.

[48] K. Kamran, "On the scintillation index of amulti wavelength Gaussian beam in a turbulent free-spaceoptical communications channel," Journal of the Optical Society of America A, vol. 23, no 3, pp. 557-566, 2006, doi: 10.1364/JOSAA.23.000557.

[49] A. Richard, "Atmospheric turbulence effects mitigation for a ground to geostationary satellite optical link: impact on the ground terminal architecture," $\mathrm{PhD}$ dissertation, Opt Phot, Ssaclay.Univ, Paris, France, 2017. [Online]. Available: https://tel.archives-ouvertes.fr/tel-02185130/.

[50] B. S. Robinson, D. M. Boroson, D. A. Burianek, D. V. Murphy, "The lunar laser communications demonstration," International Conference on Space Optical Systems and Applications ICSOS, 2011, pp. 54-57, doi: 10.1109/ICSOS.2011.5783709.

[51] M. Safarabadi, H. Izi, J. Haghshenas, H. Koochaki Kelardehc "Design of micro-vibration isolation system for a remote-sensing satellite payload using viscoelastic Materials" Engineering Solid Mechanics, Vol. 8, Issue 1, pp. 6976, 2020, doi: 10.5267/j.esm.2019.8.003

[52] H. Chen, et al, "A shared local oscillator spatial diversity PM-CO-OFDM systems based on group timing synchronization and diversity branch phase correction in satellite-to-ground optical communications," Optics Communications, vol. 479, ID. 126468, 2021, doi: 10.1016/j.optcom.2020.126468.

[53] A. U. Chaudhry, H. Yanikomeroglu, "Free Space Optics for Next-Generation Satellite Networks," IEEE Consumer Electronics Magazine, 2020, doi: 10.1109/MCE.2020.3029772. 